\section{Cahiers de Narratologie}

Analyse et théorie narratives

$11 \mid 2004$

Figures de la lecture et du lecteur

\title{
Du cinéphile au vidéophage : naissance d'un nouveau spectateur
}

Jean-Paul Aubert

\section{OpenEdition}

Journals

Édition électronique

URL : http://journals.openedition.org/narratologie/3

DOI : 10.4000/narratologie.3

ISSN : 1765-307X

Éditeur

LIRCES

Référence électronique

Jean-Paul Aubert, «Du cinéphile au vidéophage : naissance d'un nouveau spectateur », Cahiers de Narratologie [En ligne], 11 | 2004, mis en ligne le 10 janvier 2011, consulté le 30 avril 2019. URL : http:// journals.openedition.org/narratologie/3 ; DOI : 10.4000/narratologie.3

Ce document a été généré automatiquement le 30 avril 2019

Article L.111-1 du Code de la propriété intellectuelle. 


\title{
Du cinéphile au vidéophage : naissance d'un nouveau spectateur
}

\author{
Jean-Paul Aubert
}

Plusieurs articles et ouvrages récents ont cherché à brosser le portrait d'un «nouveau spectateur » qui attend du cinéma non pas qu'il lui raconte une histoire, encore moins qu'il lui propose une explication rationnelle du monde, mais plutôt qu'il lui procure des sensations inédites. Dans un article précurseur intitulé « Du spectateur fictionnalisant au nouveau spectateur: approche sémiopragmatique ", publié en 1988 dans la revue Iris, Roger Odin évoquait déjà un récepteur « qui ne vibre plus tant aux événements racontés (effets fiction) qu'aux variations de rythme, d'intensité et de couleur des images et des sons $»^{1}$. De nombreux films ont, depuis lors, confirmé l'émergence de ce nouveau spectateur. Aux Aventuriers de l'arche perdue, à La Guerre des étoiles ou à la série des Mad Max , qu'évoquait Roger Odin, sont venus s'ajouter d'innombrables films destinés à offrir toujours plus d'émotion, toujours plus de vertige. Pour Laurent Jullier, auteur d'un remarquable essai consacré à ce qu'il nomme des "films concert », certains créateurs " aspirent à court-circuiter l'intellect du spectateur pour toucher "directement " son système sensoriel $»^{2}$. Et de citer des œuvres telles Le Grand bleu, Terminator, Nirvana ou Taxi , qui ont en commun de privilégier la dimension sonore du spectacle cinématographique ainsi que des figures visuelles récurrentes comme le travelling-avant afin de s'assurer une réception essentiellement émotionnelle.

La tentation est grande de voir dans ce cinéma, parfois associé, du fait des sensations vertigineuses qu'il procure, au tour de manège ou au feu d'artifice, un adversaire du cinéma narratif traditionnel basé sur des intrigues, parfois complexes, et réclamant du spectateur une véritable collaboration intellectuelle. Cependant, loin de s'épuiser, le cinéma narratif semble, en fait, montrer sa capacité d'intégrer les apports du cinéma sensoriel. Le fait est que de nombreux films cherchent à concilier une forme classique de récit (où, pour simplifier, l'ordonnancement des séquences découle d'une vision logique fondée sur la relation de cause à effet ${ }^{3}$ ) et une approche qui fait la part belle aux stimuli audiovisuels et aux perceptions sensitives. Un cinéaste comme Vicente Aranda parvient à 
concilier un récit de facture classique avec une mise en image inédite du corps, conçu comme un médiateur de sensation, et s'adressant à la kinesthésie du spectateur ${ }^{4}$.

Il n'en demeure pas moins que, comme l'ont souligné plusieurs critiques, ce cinéma semble résister -au moins en partie-- à une analyse textuelle. Pour Laurent Jullier, « les chercheurs, qui avaient jusque-là conçu des outils permettant d'étudier la réception spectatorielle au seul stade des mécanismes descendants (sémiologie, narratologie), sont donc aujourd'hui mis en demeure d'en trouver de nouveaux à moins de voir un pan entier de la production cinématographique contemporaine leur échapper $»^{5}$. Cette remarque ouvre donc la voie à une exploration non exclusivement textuelle de l'image cinématographique.

\section{Une approche culturelle du cinéma}

4 Ce qu'il est convenu d'appeler les recherches culturelles ou cultural studies offrent à cet égard des perspectives que l'on ne saurait négliger. Il convient d'en rappeler brièvement le cadre théorique. Chacun sait qu'en envisageant le texte poétique dans sa dimension d'acte de communication, l'Ecole de Constance, à l'origine de l'esthétique de la réception, a permis de dépasser une conception strictement immanentiste héritée du structuralisme. On connaît les deux versants de l'esthétique de la réception : l'un, incarné par W. Iser, envisage l'acte de lecture dans une perspective phénoménologique et individuelle tandis, que l'autre, représenté par H.-R. Jauss s'intéresse à l'herméneutique de la réception publique de l'œuvre, dans une perspective historiciste ${ }^{6}$. Pour Paul Ricoeur, loin de s'opposer, ces deux démarches sont complémentaires :

5 En fait, elles se présupposent mutuellement: c'est d'une part, à travers le procès individuel de lecture que le texte révèle sa «structure d'appel »; d'autre part, c'est dans la mesure où le lecteur participe aux attentes sédimentées dans le public qu'il est constitué en lecteur compétent ; l'acte de lecture devient ainsi un chaînon dans l'histoire de la réception d'une œuvre par le public. L'histoire littéraire, rénovée par l'esthétique de la réception, peut ainsi prétendre inclure la phénoménologie de l'acte de lire ${ }^{7}$.

6 L'acte de lecture est, en quelque sorte le produit d'une «tension dialectique » entre un lecteur individuel et subjectif que privilégie l'analyse d'Yser et une communauté des lecteurs que souhaite cerner Jauss.

7 Cette mise au point théorique invite à une réflexion sur la manière dont s'opère la rencontre entre "le monde du texte » et "le monde du lecteur ", pour reprendre deux expressions dues à Paul Ricoeur. C'est dans cette perspective que s'inscrivent les travaux de Roger Chartier qui entendent prendre en compte « l'historicité des modes d'utilisation, de compréhension et d'appropriation des textes $»^{8}$. Sa démarche a, comme chacun sait, inspiré un certain nombre de travaux récemment menés sur le concept de "public ", d'une part, et sur les formes matérielles d'appréhension et de diffusion du texte, d'autre part. Ainsi Florence Dupont étudie-t-elle la lecture à Rome en liant la production et la réception des textes à leurs contextes culturels ${ }^{9}$. Hélène Merlin s'efforce, quant à elle, de réfléchir à la notion de "public » en France au XVIIe siècle ${ }^{10}$. Dans un ouvrage récent, Christian Vandendorpe résumait ainsi la pensée qui domine ces travaux :

8 Comme le texte n'existe qu'en fonction de la lecture, les mutations du premier ont des répercussions sur la seconde, de même que celles de la seconde entraînent nécessairement la mise en place d'un autre mode de textualité ${ }^{11}$. 
9 L'analyse esthétique de l'image cinématographique ne peut, à son tour, ignorer les conditions matérielles de diffusion et de réception de cet objet particulier qu'est le film. C'est ainsi que dans un travail publié il y a peu, Raphaëlle Moine émet l'hypothèse selon laquelle la nouvelle cinéphilie que génère le cinéma post-moderne ou qui la sous-tend n'est pas dissociable de nouvelles pratiques culturelles liées à la diversification des supports d'images (la vidéo, le DVD ou Internet) ${ }^{12}$. Deux méthodes s'offrent à celui qui entend objectiver le rapport du spectateur à l'acte qui consiste à regarder un film. La première analyse les discours du spectateur lorsqu'il se fait commentateur de son acte de visionnage. Il n'est pas rare que des travaux portant sur la réception prennent pour corpus d'étude les articles critiques publiés dans la presse, qu'elle soit spécialisée ou non ${ }^{13}$. Ainsi que le suggérait Raphaëlle Moine, il conviendrait d'envisager d'étendre ces enquêtes aux textes rédigés sur des sites personnels ou à l'occasion de forums animés par les internautes cinéphiles. Ce mode d'expression connaît en effet un développement fulgurant grâce notamment à la multiplication de sites Internet sur le modèle du célèbre UKMDB (United Kingdom Movie data base) qui engagent les visiteurs à se faire critiques de cinéma. Ces recherches seraient d'autant plus précieuses qu'elles porteraient sur la réception des «films concerts » dont Raphaëlle Moine constate qu'elle est indissociable d'une pratique assidue des outils informatiques. Selon une démarche comparable, on peut envisager une étude du public sous forme d'enquête menée à la sortie des salles de cinéma. On sait que certains producteurs soumettent déjà, à des fins commerciales, des échantillons du film en préparation à un panel de spectateurs que l'on suppose représentatif. L'objectif étant, on le comprend aisément, de faire évoluer le produit cinématographique en fonction des réactions des spectateurs cobayes. En dépit de l'intérêt évident qu'est susceptible de présenter une telle approche, on ne peut en ignorer les limites. Bourdieu, notamment, rappelle les difficultés inhérentes à l'évaluation de ces enquêtes du fait de ce qu'il nomme l' " effet de légitimité » ${ }^{14}$.

La seconde approche, complémentaire de celle que nous venons d'évoquer rapidement, consiste à réinterroger l'objet film lui-même afin d'examiner de quelle façon sa structure détermine un protocole de lecture. C'est cette approche que nous entendons privilégier dans le cadre de ce travail qui tentera d'appliquer au cinéma la distinction qu'opère Roger Chartier pour la littérature entre "mise en livre» et "mise en texte». En d'autres termes, nous nous intéresserons au moins autant au dispositif (autrement dit les formes matérielles de transmission et d'appréhension des images cinématographiques) qu’à l'écriture elle-même.

11 Nul n'ignore que les années soixante-dix et quatre-vingt ont été marquées par l'arrivée d'un nouveau vecteur d'images : la vidéo. On ne peut nier aujourd'hui que l'irruption de cette nouvelle technologie a constitué une révolution des structures matérielles de la représentation cinématographique. Quelques chiffres suffisent à dire l'ampleur du phénomène. Selon le Centre National de la Cinématographie, en 1999, les 4764 salles de cinéma en fonction sur le territoire français ont accueilli quelque 155 millions de spectateurs. Au cours de cette même année, on estime à environ dix millions le nombre de films en vidéos achetées et à 120 millions le nombre de personnes qui les ont regardées. Il s'agit des résultats d'un essor rapide de ce nouveau support qui a facilité la rencontre entre les films et le public cinéphile et a parfois permis à ce dernier de découvrir ou de redécouvrir des continents cinématographiques oubliés. Ainsi le support vidéo a-t-il apporté un démenti à la prédiction pessimiste de François Truffaut qui s'attristait de ce que dans l'avenir des films puissent être réalisés et surtout critiqués par 
des gens qui n'auraient jamais vu L'Aurore de Murnau. Dans le même temps, la vidéo a transformé notablement les modalités de réception de l'image. Certains l'ont regretté, s'inquiétant de la concurrence qu'elle opposait aux salles. Cependant, il ne s'agira pas pour nous d'entrer dans cette querelle des anciens et des modernes, qui oppose les tenants irréductibles du grand écran aux partisans de la vidéo. En revanche, c'est à l'évolution des habitudes spectatorielles que nous voulons consacrer le premier temps de notre réflexion.

Si l'on en croit les chiffres énoncés précédemment, on doit admettre que l'on est en présence d'une génération de spectateurs qui doit sa culture cinéphile au moins autant au visionnage de cassettes vidéos qu'à la fréquentation des salles d'art et d'essai, des cinéclubs ou des cinémathèques. Or, c'est parmi ces cinéphiles devenus vidéophages que se recrutent les nouveaux cinéastes. De la même façon que l'on a pu dire de la Nouvelle vague et, plus largement, des nouveaux cinémas qu'ils étaient, en partie, les produits du mouvement des ciné-clubs et de l'engagement en faveur des cinémathèques, il convient de s'interroger sur l'influence qu'un nouveau vecteur d'images tel que la vidéo a pu exercer sur l'esthétique du cinéma d'aujourd'hui. Cette interrogation sera donc au cœur de la seconde partie d'une contribution qui aurait pu avoir en exergue ces mots de Régis Debray : « Le support est peut-être ce qui se voit le moins et ce qui compte le plus » ${ }^{15}$.

Du celluloïd à la vidéo

Et si le cinéma avait réalisé en moins d'un siècle ce que la littérature mit des millénaires à accomplir ? À bien y regarder, la position du spectateur de cinéma traditionnel reproduit celle du public des conteurs et des troubadours. Les trois niveaux de contraintes que Christian Vandendorpe énumère lorsqu'il évoque l'auditeur d'antan sont ceux qui s'imposent au public des salles de cinéma :

- a) l'auditeur n'a pas la possibilité de déterminer le moment de la communication ;

- b) il n'en maîtrise pas le débit, prisonnier qu'il est du rythme choisi par le conteur ;

- c) en matière d'accès au contenu, il n'a aucune possibilité de retourner en arrière afin de sélectionner, dans un récit déjà connu, la séquence qui l'intéresse particulièrement : il doit suivre le fil, irrémédiablement linéaire parce qu'inscrit dans le temps, de la récitation qui en est faite ${ }^{16}$.

Si l'on accepte cette parenté entre l'auditeur de récit oralisé et le spectateur installé dans une salle de cinéma, on peut admettre que l'apparition du support vidéo soit comparée à la naissance de l'écriture et de la lecture. De la même façon que l'invention de l'écriture va modifier les relations du récepteur à l'œuvre, l'essor et la généralisation du support vidéo transforment les rapports entre les films et leurs spectateurs. Comme le livre le fait pour le texte, la cassette VHS a vocation à stocker des images que le spectateur peut consommer à sa guise. Sur un plan socioculturel, on constate là encore pour le cinéma une évolution semblable à celle que connit le livre, la consommation de l'image relevant désormais de la sphère du privé. Mais la grande nouveauté réside dans la disparition des contraintes de programmation des salles (des contraintes que l'apparition de la télévision n'a pas fait disparaitre) et, par conséquent, dans le libre accès du public à l'image cinématographique. Enfin, le support vidéo et le magnétoscope ont créé un nouveau type de consommateurs d'images doté d'un pouvoir dont le spectateur soumis aux conditions de projections traditionnelles ne disposait pas. Avec l'arrêt sur image, l'avance rapide ou le ralenti, c'est bien la possibilité de manipuler le récit, de le triturer et pourquoi pas de le détruire, qui se fait jour. À cet égard, l'on s'aperçoit désormais que la véritable révolution ne résidait pas tant dans la réduction de l'écran (autrement dit le passage du grand écran 
de la salle de cinéma au petit écran du téléviseur) que dans l'invention d'un petit objet, la télécommande, instrument d'une véritable lecture du film. Régis Debray avait clairement perçu le changement qu'allait introduire cet accessoire dans la réception des images. Pensons par exemple à tout ce que la télécommande, minuscule innovation technique, est en train de changer dans notre regard, et dans notre culture même: discontinuité, juxtaposition, culte du temps fort. Voilà une autre façon de circuler non dans mais entre les images. Qui fait son marché au petit écran, s'habitue à rentrer dans le récit par n'importe quel bout ${ }^{17}$.

16 La commande du magnétoscope devient un scalpel qui prélève des instants du récit pour les soumettre à l'analyse et en révéler la valeur émotionnelle propre. Le film a acquis une matérialité dont était dépourvu le faisceau lumineux issu du projecteur de la salle de cinéma. Le flux cinématographique peut être paralysé, décomposé jusqu'à n'être plus qu'une succession de photographies, de tableaux, d'instants. Le support DVD n'a fait que perfectionner ce que la vidéo autorisait déjà. Afin de lui faciliter la tâche, les DVD s'efforcent, en effet, de proposer au spectateur une indexation des séquences (ou chapitrage) qui lui permet de se caler directement et sans tâtonnement sur la séquence qu'il souhaite visionner. Une fois encore la comparaison avec l'histoire du livre semble s'imposer. De la même façon que le découpage du texte en chapitres et l'ajout d'une table des matières permettaient une lecture individualisée et parcellaire, la structure même de l'objet DVD autorise, et encourage même une lecture discontinue du film. L'enjeu ne se situe plus désormais dans une lecture du récit qui serait tournée vers la résolution d'une tension liée à l'intrigue. Il réside davantage dans l'aptitude offerte au vidéophage de choisir dans le flux d'images, celles dont la plasticité est la plus apte à susciter en lui des émotions. De sorte que l'usage du magnétoscope ou du lecteur de DVD est de nature à favoriser une appréhension de l'image presque exclusivement tournée vers la jouissance.

Par ailleurs, il est probable que la vision magnétoscopée est à l'origine d'un renouveau du regard porté sur les films et sur le cinéma d'une manière générale ; un renouveau comparable à bien des égards à la révision de l'histoire de l'art que provoqua la possibilité de photographier les tableaux. Très vite, théoriciens et praticiens du cinéma ont perçu la vidéo comme l'outil permettant une réécriture de l'histoire du cinéma par la comparaison effective des œuvres et par l'étude plus attentive de l'écriture filmique. Le support vidéo non seulement autorise, mais véritablement invite à une analyse de la plasticité du film qui pouvait échapper au spectateur soumis aux conditions de projections traditionnelles. La capacité offerte à ce dernier d'altérer la continuité du récit, de détacher des instants du continuum spatio-temporel a probablement contribué à ce que la valeur narrative du film prenne un caractère presque secondaire au regard de sa dimension esthétique. La multiplication de travaux portant sur la représentation du corps filmé s'inscrit, du reste, dans cette perspective. L'évolution des corps à la surface de l'écran prend d'autant plus aisément l'apparence d'une chorégraphie que la télécommande nourrit l'illusion d'une possible maîtrise des mouvements. L'arrêt sur image, l'accéléré, la marche arrière le morcellent, soumettent le corps à un examen clinique non sans exercer une certaine forme de violence à son encontre. Une étude récente de Dominique Païni publiée dans Les cahiers du cinéma associait, précisément, l'essor du support vidéo à l'émergence d'une nouvelle cinéphilie, moins soucieuse de la virtuosité du récit que de la plasticité de l'œuvre, dotée d'un regard avant tout chorégraphique, sensible à la dynamique des corps et à l'expression d'une corporéité retrouvée, une cinéphilie -écrivait-il- « qui revalorise la dimension figurative des corps, leur brusque métamorphose, l'autonomisation du geste et 
de l'action, le jeu alternatif de la présence et de la disparition, les vitesses, la valeur plastique du mouvement $»^{18}$. Ainsi, grâce à la vidéo, le spectateur redécouvrirait-il les obsessions d'un Etienne-Jules Marey scrutant le mouvement et la chorégraphie des corps.

\section{Vers une esthétique de la fragmentation}

18 On l'aura compris, le second volet de cette réflexion concerne les stratégies d'écriture cinématographique que la généralisation de ces nouveaux supports est susceptible de favoriser. Disons d'emblée que la recherche d'une corrélation entre l'émergence d'un nouveau dispositif et l'adoption de nouvelles formes esthétiques doit être envisagée avec la plus extrême prudence, d'autant que le support vidéo ne se substitue jamais totalement au dispositif spectatoriel traditionnel. Les deux modalités d'accès à l'image que sont la salle de cinéma et le magnétoscope coexistent et sont perçues tant par les professionnels que par le public comme complémentaires. Cela signifie que, dans leur immense majorité, les films n'ont pas été conçus pour une diffusion exclusive sous forme de cassette VHS ou de $\mathrm{DVD}^{19}$. Alors que la télévision a engendré des productions audiovisuelles spécifiques, rares, en effet, sont les œuvres exclusivement destinées au support vidéo. La contrainte que ce type de diffusion est susceptible d'exercer sur le discours des réalisateurs ne peut donc s'affranchir totalement d'une autre contrainte: celle qui est liée à la pérennité, jusqu'à nouvel ordre, des salles de cinéma. Le film, destiné à la fois à être projeté en salle et reproduit sous forme de vidéo, visera donc à satisfaire un public multiple, autant cinéphile que vidéophage. Cette nuance étant apportée, devrions-nous nous étonner de ce que l'esthétique mise en œuvre par un certain nombre de films récents soit considérablement influencée par l'usage du magnétoscope quand leurs auteurs euxmêmes reconnaissent qu'elle leur est familière? Alejandro Amenábar revendique cette culture acquise grâce à la vidéo :

J'ai commencé à voir des films en vidéo, avec mon frère, chez un couple d'Américains, voisins et amis de mes parents. Après les avoir vus, je les recomposais mentalement ce qui m'aida beaucoup. Presque sans m'en rendre compte, j'analysais les films, pensais à certains plans et découvrais comment ils étaient faits. Ensuite, pendant la récréation, je les racontais à mes camarades de collège en laissant libre cours à mon imagination. Entre neuf et treize ans, je voyais presque tous les films en vidéo $[\ldots]^{20}$.

Il en va de même de son jeune compatriote, Alex de la Iglesia :

Pour ma part, j'ai découvert le cinéma par la télévision, et cela symbolise un peu ma façon de voir les choses. Le petit écran offre un étalage chaotique et désordonné sans critère régulateur, ni ordre chronologique $[. . .]^{21}$.

Avec ces jeunes auteurs, on a donc clairement affaire à une nouvelle génération de cinéastes dont la culture cinéphile est indissociable de l'apparition et de l'essor du support vidéo. Les propos d'Alejandro Amenábar et d'Alex de la Iglesia nous semblent donc de nature à justifier une interrogation que l'on pourrait formuler en ces termes : dans quelle mesure l'écriture filmique prend-elle en compte les protocoles de lectures que nous venons de décrire?

21 La mise en scène par le cinéma de ces nouvelles pratiques et la fictionnalisation répétée de spectateurs vidéophages apparaissent comme une première réponse. Ne constituent-telles pas, en effet, la reconnaissance par les auteurs eux-mêmes d'une modalité de réception de leurs œuvres sinon souhaitée du moins envisagée? 
Dans l'une des premières séquences du film Tesis (Alejandro Amenábar, 1996), le personnage principal, une jeune étudiante en sciences de l'audiovisuel, prénommée Angela, visionne pour sa thèse, la vidéo d'un film gore. Est-ce un hasard si l'actrice qui lui prête vie est celle qui vingt-cinq ans plus tôt avait incarné Ana, l'une des deux fillettes du film de Victor Erice, El espíritu de la colmena ? Le regard d'Angela reflète une frayeur que les grands yeux noirs d'Ana avaient déjà exprimée un quart de siècle auparavant tandis qu'ils découvraient sur l'écran d'une modeste salle de cinéma d'un petit village de Castille les images de Frankenstein. Du reste le spectacle du film gore n'est pas moins terrifiant que celui de Frankenstein et la créature démoniaque s'est muée en un personnage tout aussi malfaisant. Cependant, si la permanence du regard grave d'Ana Torrent semble vouloir nous faire admettre que la diminution de la taille des images n'amoindrit pas leur effet, elle ne peut faire oublier l'évolution du dispositif spectatoriel. L'écran a changé de taille et le rituel du cinéma que décrit la première séquence de El espíritu de la colmena s'est changé en de nouvelles habitudes qu'Alejandro Amenábar enregistre en sociologue. Il n'est pas le seul à faire le constat de l'évolution des pratiques cinéphiles. La prise de conscience des multiples voies d'accès aux images qu'offre la société moderne et la reconnaissance de la concurrence qu'opposent à la salle de cinéma la vidéo, l'écran de l'ordinateur ou Internet sont des constantes de la production cinématographique récente. Il suffit, pour s'en convaincre, de revoir des films tels que Las edades de Lulú (1990) de Bigas Luna, Historias del Kronen (1995) de Montxo Armendáriz, La mirada del otro (1997) de Vicente Aranda ou Nadie conoce a nadie (1999) de Mateo Gil.

On se souvient que dans les films des années cinquante ou soixante, il n'était pas rare que le protagoniste trouve un instant de répit dans une salle de cinéma. Les cinéastes de la nouvelle vague multiplièrent ces séquences qui étaient autant d'occasions de rendre hommage à l'auteur dont on projetait le film et plus largement au cinéma en tant qu'art populaire. Peut-être est-il exagéré de déduire des exemples cités précédemment que les personnages des films d'aujourd'hui ne fréquentent plus guère les salles obscures et, qu'à l'image de leurs contemporains de chair et d'os, ils leur préfèrent la consommation de produits vidéo ou la visite de sites Internet. Il n'en demeure pas moins que les films que nous venons d'évoquer semblent prendre acte de l'évolution des pratiques culturelles et font de leurs personnages des consommateurs d'images auxquels le spectateur contemporain peut, dans une certaine mesure, s'identifier.

En mettant en scène un nouveau spectateur, ces longs-métrages ne manquent pas de s'interroger sur la nature de cet objet particulier qu'est le film, à l'heure où l'écran de cinéma n'est plus que l'un des multiples supports susceptibles de l'accueillir. Ils engagent, à n'en pas douter, une redéfinition du cinéma et n'hésitent pas à remettre en cause les frontières dans lesquelles ce dernier s'était jusqu'alors cantonné. Ils entendent également questionner le regard particulier sur l'image que suppose l'usage du magnétoscope. Il est frappant de constater que tant Tesis que Historias del Kronen ou Las edades de Lulú associent la visualisation de l'image vidéo à une quête effrénée de sensations extrêmes. Dans Tesis, la protagoniste découvre la pratique clandestine des snuff movies à laquelle se livre un groupe d'étudiants. Historias del Kronen brosse le portrait d'un groupe de jeunes désœuvrés qui font de la transgression des interdits un principe de vie. Las edades de Lulú décrit l'itinéraire hédoniste d'une jeune femme qui multiplie les expériences érotiques extrêmes. Pour tous ces personnages, la vidéo, au même titre que les drogues, la musique, la vitesse, le sexe, ne semble avoir d'autre fonction que celle de les plonger dans un état qui est de l'ordre du sensoriel et de l'infra intellectuel, en provoquant une forme 
d'étourdissement, de perte de soi, d'ivresse sans laquelle il n'est pas de grand frisson. La protagoniste féminine de Las edades de Lulú dit avoir éprouvé une émotion sexuelle particulière lors du visionnage de vidéos pornographiques. Dans le même ordre d'idée, une séquence de Historias del Kronen montre Carlos et Roberto visionnant une vidéo qu'ils rembobinent frénétiquement afin de revoir une seule et unique séquence dont la violence les excite. Le visionnage en boucle est, à cet égard, symptomatique d'un désir inconscient de renouer avec le plaisir infantile et primitif de la répétition. C'est dire si l'on retrouve à travers la mise en scène du consommateur de vidéos que ces films proposent, l'évocation du spectateur de l'extrême que nous décrivaient Laurent Jullier et Roger Odin, un spectateur avide de sensations, prêt à s'abandonner à l'ivresse que lui procurent les images et les sons.

La fictionnalisation de ce nouveau spectateur vidéophage peut donc être perçue comme l'indice d'une prise en compte des enjeux esthétiques et éthiques liés à la généralisation du support vidéo. Il n'est pas le seul. On ne peut ignorer le fait que l'écriture cinématographique tend de plus en plus vers une esthétique de la fragmentation, qui semble faire écho à la manipulation et à la parcellisation de l'image et au morcellement du récit filmique qu'autorise désormais l'utilisation généralisée de la vidéo. Dans les films considérés ici, cette esthétique de la fragmentation est décelable à plusieurs niveaux dont celui du montage, celui de la texture de l'image et celui enfin de l'évocation ou de la représentation du corps.

Dans les dernières séquences du film Tesis d'Alejandro Amenábar, Angela, prise au piège, se retrouve face au tortionnaire et auteur des snuff movies. D'une manière assez générale, le montage privilégie une succession de plans de courte durée aboutissant à une dispersion de l'espace que viennent conforter les choix réalisés en matière de taille des plans et de profondeur de champ. Alors que les plans d'ensemble permettraient au spectateur d'embrasser l'espace dans sa globalité, les plans rapprochés et gros plans qui abondent dans le film, produisent la sensation inverse d'un fractionnement. La réduction de la profondeur de champ complète le dispositif. L'utilisation fréquente de téléobjectifs a pour double résultat d'enserrer le sujet dans un cadre extrêmement réduit et d'estomper l'arrière-plan ${ }^{22}$. Les personnages se dessinent sur un fond volontairement flou et indistinct, de sorte qu'ils apparaissent comme détachés d'un univers qui tend vers l'abstraction. L'appréhension de l'espace dans lequel évoluent les personnages, si tant est qu'il est possible, exige alors du spectateur un effort de reconstruction mentale.

Certes, le resserrement du cadrage et l'absence de profondeur de champ expriment formellement l'oppression que subit le personnage d'Angela. Mais il est difficile de ne pas apprécier un tel travail sur l'échelle des plans et sur la profondeur de champ au regard des conditions particulières de visionnage qu'imposent le magnétoscope et l'écran de télévision. Ramenés aux dimensions d'un petit écran, les plans d'ensemble perdent de leur intensité. De fait ils sont pour ainsi dire proscrits du film dont il est question ici, au profit des plans rapprochés et des gros plans comme si son auteur, consciemment ou inconsciemment, avait formaté l'image par avance, selon des normes appropriées au visionnage sur écran de télévision. La réduction de la profondeur de champ est également en adéquation avec les contraintes du petit écran dont chacun sait qu'il en atténue considérablement les effets. Là encore, le choix des optiques semble anticiper le format du petit écran.

Mais cette élaboration des plans n'est pas qu'une adaptation technique aux conditions matérielles de la vision sur l'écran de télévision. En effet, on remarque qu'elle s'inscrit 
parfaitement dans le cadre de cette esthétique de la fragmentation mise en œuvre par le montage. Un montage qui cultive la discontinuité en juxtaposant des images aux textures les plus variées. Ainsi les deux dernières séquences de Tesis associent-elles en une sorte de collage audiovisuel des images tournées avec une caméra traditionnelle à d'autres, réalisées au moyen d'une caméra vidéo, et à d'autres encore qui imitent le générique en noir et blanc d'une émission de télévision. Cette hétérogénéité est une caractéristique de Tesis mais aussi d'Historias del Kronen dont la dernière partie est dominée par des images tournées à l'aide d'une caméra vidéo. À ce mélange d'images d'origines diverses s'ajoute, parfois, une altération du flux filmique. C'est ainsi que le ralenti ou l'accélération, deux procédés utilisés par Alejandro Amenábar dans ces dernières séquences de Tesis, semblent devancer, voire légitimer, une lecture magnétoscopée.

Cette image à la fois fragmentée et triturée est tout particulièrement adaptée au discours sur le corps que la plupart de ces films véhiculent. De fait, la déstructuration de l'image est une mise en danger du corps. Elle suggère une remise en cause de son unité. Le ralenti ou l'accéléré déréalisent le mouvement corporel et participent d'une mécanisation dépersonnalisante du personnage ${ }^{23}$.

Dans Las edades de Lulú, de Bigas Luna, le détail envahit l'écran, le corps devient pure abstraction. Son homogénéité rassurante disparait au profit d'une parcellisation qui dérange. L'insert crée une étrange autonomie des parties par rapport au tout, ne retenant de l'anatomie qu'un fragment d'épiderme. L'arrondi d'un sein, le galbe des hanches, la béance d'un sexe dessinent alors un paysage presque abstrait.

31 Il n'est pas rare que cette fragmentation du corps soit la traduction esthétique d'une violence commise à son égard qui, lorsqu'elle n'est pas littérale, s'exprime, sans détours, dans les dialogues. Dans Tesis, la description que fait Bosco du supplice qu'il prévoit d'infliger à sa victime est, à cet égard, tout à fait significative :

Pour commencer, je vais te cogner sur la tête pendant... un certain temps. Une fois que t'as perdu connaissance, je te laisse récupérer. Ensuite, je t'écorche les bras et les jambes. Puis, je te mutile. Quoi, je ne sais pas encore. Ce genre de choses, je les improvise. Si par exemple, je te coupe une main, je peux te la poser sur la tête. Comme si c'était un diadème. Ces conneries, ça fait son effet. Je peux faire en sorte que tu manges ta propre oreille. Et même, si ça me passe par la tête, je peux t'extraire les boyaux sans que tu meures. Ça fout les jetons, hein ? [...] Une fois que tu as perdu beaucoup de sang et que tu es presque morte, je te tire une balle dans la bouche. Ensuite, je te découpe en morceaux [... ${ }^{24}$.

Une fois de plus, on ne peut que remarquer l'étonnante isomorphie qui se fait jour entre le dispositif de visionnage des films et l'évocation ou la représentation de ce corps morcelé, décomposé, parcellisé, que la cinématographie la plus récente ne cesse de reproduire.

La fragmentation qui s'exerce au niveau du montage ou de l'élaboration plastique des plans affecte, en dernier lieu, la narration. Certes, les films évoqués ici ne renoncent pas totalement au récit et notamment aux structures causales qui le fondent. Mais la trame devient minimale. La découverte de l'univers malsain des snuff movies dans le cas de Tesis ou la quête de sensations toujours plus fortes, dans les cas d'Historias del Kronen, de Las edades de Lulú ou de La mirada del otro servent en fait de prétexte pour permettre aux protagonistes d'accomplir un parcours aléatoire dont les principaux jalons sont autant d'expériences nouvelles. L'essentiel n'est plus dans le récit mais dans l'espace poétique créé par la chorégraphie des corps et la plasticité des images ${ }^{25}$. On devine chez ces auteurs la volonté de créer un langage qui déborde le narratif et qui s'adresse d'autant mieux aux 
amateurs de vidéo qu'il travaille la substance filmique, juxtapose les plans et les séquences comme autant d'instants ou de temps forts, comme le ferait le vidéophage, armé de sa télécommande. Les films, comme s'ils prenaient acte de la liberté nouvelle du spectateur décrivent un espace qu'il est libre de parcourir. Il peut y flâner à sa guise, se laisser séduire par un plan, s'émouvoir du choc improbable de deux images.

\section{Conclusion}

Certes, il ne s'agit pas d'expliquer l'attention portée par le cinéma actuel à la représentation du corps, son goût pour la déstructuration du récit ou sa prise en compte de la matérialité de l'image par la seule apparition du support vidéo. Ce serait succomber à un déterminisme naïf. On sait du reste qu'au-delà du septième art, ces caractéristiques s'inscrivent dans une tendance générale des pratiques artistiques actuelles au point que l'on a pu dire de la fragmentation qu'elle était véritablement devenue l'un des signes distinctifs de l'art contemporain. Mais l'on devine qu'au sein de ce que certains nomment déjà une « culture de la fragmentation $»^{26}$, le magnétoscope ou le lecteur de DVD occupent toute leur place.

D'une part, le support vidéo a donné à l'image cinématographique une matérialité dont elle était probablement dépourvue auparavant. Elle est devenue, pour le vidéophage, un objet concret, qu'il collectionne et qu'il échange, une réalité physique, une substance susceptible d'être altérée, trafiquée et déformée. Il n'est pas interdit de penser que les divers procédés mis en place par la nouvelle génération de cinéastes et qui s'appliquent à retravailler la substance même de l'image soient le fruit d'une prise de conscience de la matérialité du film qui a été encouragée par l'utilisation assidue de la vidéo.

D'autre part, la capacité qui est offerte au vidéophage d'altérer la continuité du récit, de détacher des instants du continuum spatio-temporel a probablement contribué à ce que la valeur narrative du film prenne un caractère presque secondaire au regard de sa dimension esthétique avec comme conséquence l'attention renouvelée du spectateur à l'égard de la représentation du corps. C'est, à coup sûr, ce regard nouveau du magnétoscopeur qui s'affirme dans les films d'Alejandro Amenábar ou d'Alex de la Iglesia tout comme il perce déjà dans les essais précurseurs de Jean-Luc Godard tels que On s'est tous défilé (1988) ou Sauve qui peut (la vie $)^{27}$, ou comme il triomphe dans les œuvres cinématographiques d'Atom Egoyan ou de David Kronenberg, aboutissements de recherches esthétiques sur le mouvement des corps qui incluent la décomposition et la déconstruction.

\section{NOTES}

1. Roger Odin, «Du spectateur fictionnalisant au nouveau spectateur », Iris, Vol. $5, \mathrm{n}^{\circ} 1,2^{\mathrm{e}}$ semestre 1988, p. 134.

2. Laurent Jullier, L'Ecran post-moderne, un cinéma de l'illusion et du feu d'artifice, Paris, L'Harmattan, coll. « Champs visuels », 1997, p. 37. 
3. Rappelons que pour Edward Branigan, le récit « est une manière d'organiser les données spatiales et temporelles en chaîne d'événements liés par une relation de cause à effet, avec un début, un milieu et une fin, manière d'organiser qui matérialise un jugement sur la nature des événements aussi bien qu'elle démontre comment il est possible de connaître ces événements, et donc de les narrer » (Edward Branigan, Narrative comprehension and films, Londres \& New-York, Routledge, 1992, cité par Laurent Jullier, L'Ecran post-moderne, un cinéma de l'ilusion et du feu d'artifice, op. cit., p. 100)

4. On pourra se reporter aux travaux que nous avons consacrés à cet auteur : Le cinéma de Vicente Aranda. Pour une esthétique du personnage filmique, Paris, L'Harmattan, coll. "Champs visuels ", 2000 (voir en particulier le chapitre II, « représenter le corps ») et « Un autre regard sur le corps : La Mirada del otro de Vicente Aranda ", in Fabrice Parisot (éd.), Hommage au Professeur Gérard Lavergne, publication du Centre de Narratologie Appliquée, Université de Nice-Sophia Antipolis, 2000, pp. 15-31.

5. Laurent Jullier, L'Ecran post-moderne, un cinéma de l'ilusion et du feu d'artifice, op. cit, p. 9.

6. Wolfgang Iser, L'acte de lecture. Théorie de l'effet esthétique, Bruxelles, Pierre Mardaga, coll. "Philosophie et langage», 1985 ; Hans-Robert Jauss, Pour une esthétique de la réception, Paris, Gallimard, 1978.

7. Paul Ricoeur, Temps et récit, t. 3, Le temps raconté, Paris, Seuil, 1985, p. 304.

8. Roger Chartier, Guglielmo Cavallo (éd.), Histoire de la lecture dans le monde occidental, Paris, Seuil, 1997, p. 9.

9. Florence Dupont, Homére et Dallas, introduction a une critique anthropologique, Paris, Hachette, 1991 et Lire le théâtre latin, Paris, Armand Colin, 1998.

10. Hélène Merlin, Public et littérature en France au XVIIe siècle, Paris, Les Belles Lettres, 1994.

11. Christian Vandendorpe, Du papyrus à l'hypertexte. Essais sur les mutations du texte et de la lecture, Paris, Editions de La Découverte, 1999, p. 9.

12. Raphaëlle Moine, "Approche culturelle d'un certain cinéma post-moderne: les filmsconcerts ou le cinéma de la sensation ", in Odile Bächler, Claude Murcia, Francis Vanoye (ed.), Cinéma et audiovisuel. Nouvelles images, approches nouvelles, Paris, L'Harmattan, coll. "Champs visuels ", 2000, pp. 205-212. Sur cette même question, on lira également, avec intérêt: L. Drummond, American Dreamtime, a Cultural Analysis of Popular Movies, and Their Implications for a Science of Humanity, Lanham, Rowman \& Littlefield Publishers, 1996.

13. À titre d'exemple, on peut citer Angel Luis Hueso Montón, «Luces y sombras de una cinematografía. La difusión y recepción del cine cubano en España », in Emmanuel Larraz (ed.), Voir et lire Tomas Gutierrez Alea. La mort d'un bureaucrate, Hispanistica XX, coll. "Critiques et documents », publication de l'Université de Bourgogne, 2002, pp. 57-75.

14. "La lecture : une pratique culturelle. Débat entre Pierre Bourdieu et Roger Chartier ", in Roger Chartier (ed.), Pratiques de la lecture, (1éd., Paris, Rivage, 1985), Paris, Editions Payot, 1993, pp. 273-274.

15. Régis Debray, Cours de médiologie générale, Paris, Gallimard, 1991, p. 195.

16. Christian Vandendorpe, Du Papyrus à l'hypertexte. Essai sur les mutations du texte et de la lecture, op.cit, p. 15.

17. Régis Debray, «Vie et mort de l'image. Une histoire du regard en occident », Esprit, $\mathrm{n}^{\circ} 199$, février 1994, p. 63.

18. Dominique Païni, «Faire violence. À propos du Trafic des cassettes vidéo ", Cahiers du cinéma, n524, mai 1998, p. 94. Dominique Païni commente un échange de lettres entre critiques et théoriciens du cinéma, "enfants des années soixante", publié dans la revue Trafic: Jonathan Rosenbaum, Adrian Martin, Kent Jones, Alexander Horwat, Nicole Brenez et Raymond Bellour, "Movie mutations : correspondance avec et entre quelques enfants des années soixante ", Trafic, $\mathrm{n}^{\circ} 24$, hiver 1997, pp. 5-43. 
19. Cette situation est susceptible d'évoluer très rapidement. Pour Alexander Horwath, il ne fait guère de doute que "les grands films, aujourd'hui, [...] semblent avoir été mis en scène en prévision de leur programmation sur écran vidéo." Jonathan Rosenbaum et allii, "Movie mutations... », art. cit., p. 25.

20. «Comencé a ver películas en vídeo, junto con mi hermano, en la casa de un matrimonio americano, que eran vecinos y amigos de mis padres. Luego las recomponía mentalmente y eso me ayudó mucho. Casi sin darme cuenta, analizaba las películas, me paraba a pensar en determinados planos y descubría cómo estaban hechas. Después, en el recreo, se las contaba a mis compañeros del colegio y lo hacía de una forma imaginativa. Entre los nueve y los trece años, casi todas las películas las veía en el vídeo." Entretien avec Alejandro Amenábar, Carlos F. Heredero, Espejo de mirada. Entrevistas con nuevos directores de cine español de los años noventa, Festival de cine de Alcalá de Henares, 1997, p. 85.

21. «Es que yo he descubierto el cine a través de la televisión, y esto simboliza un poco mi forma de ver las cosas. La pequeña pantalla te ofrece un muestrario caótico y desordenado, donde no hay criterio regulador ni orden cronológico alguno. » Entretien avec Alex de la Iglesia, ibid, p. 469.

22. Lire, à ce sujet, l'entretien qu'Alejandro Amenábar a accordé à Carlos F. Herredero, in Espejo de miradas. Entrevistas con nuevos directores del cine español de los años noventa, 27 Festival de Cine de Alcalá de Henares, 1997, pp. 96-97.

23. Nous avons évoqué ce geste "dépersonnalisant» dans "Propositions pour une étude du geste dans le film de Vicente Aranda, Fanny Pelopaja (1984) », in Cahiers du Groupe de Recherches Ibériques et Ibéro-américaines de l'Université de Saint-Étienne, 1998, pp. 105-125.

24. «Primero, te voy a golpear en la cabeza durante... un rato. Luego voy a rajarte en los brazos y en las piernas. Después te mutilo. Pero no sé todavía el qué. Esas cosas las improviso sobre la marcha. Si por ejemplo, te corto una mano, puedo ponértela sobre la cabeza como si fuera una diadema. Esas mariconadas dan mucho juego. Puedo hacer que te comas tu propia oreja. Incluso si se me ocurre puedo sacarte las tripas sin que te mueras. ¿Acojona eh ? [...] Cuando te desangres y estés a punto de morir, te pego un tiro en la boca. Luego te descuartizo [...]. »

25. Vincent Amiel a consacré à ces images d'aujourd'hui plusieurs travaux. Citons notamment «Le Corps en images (de Buster Keaton à John Cassavetes), Esprit, n 199, février 1994, pp. 135-146. On retiendra, en particulier, les pages dédiées à Cassavetes (pp. 142-146).

26. Vicente Sánchez-Biosca, Una cultura de la fragmentación. Pastiche, relato y cuerpo en el cine y la televisión, Valencia, Ediciones Textos Filmoteca, 1995.

27. Au sujet des relations qu'entretient Godard avec la vidéo, lire Philippe Dubois, « Godard et la vidéo. L'image à la vitesse de la pensée. », Cahiers du cinéma, numéro spécial, Août 1991, pp. 76-77.

\section{RÉSUMÉS}

Cet article, qui se place dans la perspective ouverte par les études culturelles interroge, dans un premier temps, les habitudes spectatorielles induites par l'usage répandu des supports vidéo et DVD. Dans un second mouvement, il propose une réflexion sur l'influence que ces nouveaux vecteurs d'images exerce sur l'esthétique du cinéma d'aujourd'hui à partir des exemples qu'offrent les œuvres de réalisateurs espagnols comme Bigas Luna, Alejandro Amenábar ou Alex de la Iglesia. 
Este artículo se vale de las perspectivas abiertas por los estudios culturales para cuestionar en su primera parte las prácticas del espectador de hoy en relación con el desarrollo del video y del DVD. Luego, a partir de los ejemplos que ofrecen las obras de cineastas españoles como Bigas Luna, Alejandro Amenábar o Alex de la Iglesia, propone una reflexión sobre la influencia que ejercen los nuevos vectores de imágenes en la estética del cine actual.

INDEX

Index chronologique : XXe siècle

Index géographique : Espagne

Mots-clés : cinéma, DVD, fragmentation, spectateur, vidéo

\section{AUTEUR}

\section{JEAN-PAUL AUBERT}

Maître de conférences à l'Université de Nice-Sophia Antipolis, Jean-Paul Aubert enseigne dans le département d'études ibériques et ibéro-américaines. Ses travaux portent sur le cinéma espagnol. Il est l'auteur d'une quinzaine d'articles publiés dans des revues universitaires et d'un ouvrage paru en 2001 aux éditions L'Harmattan: Le cinéma de Vicente Aranda. Pour une esthétique du personnage filmique. Il est rattaché au CNA (Centre de Narratologie Appliquée de l'Université de Nice). 